Review Article

\title{
The Impact of Negative Emotions on Switching Decisions: A Sample of Student at the University of Labour and Social Affairs, Ho Chi Minh Campus
}

\author{
Bui Hoang Ngoc* \\ Graduated School, Ho Chi Minh City Open University, \\ 97 Vo Van Tan Street, Ward 6, District 3, Ho Chi Minh City, Vietnam \\ Received 02 February 2020 \\ Revised 06 April 2020; Accepted 08 April 2020
}

\begin{abstract}
Negative emotions are the result of unpleasant experiences when using a product or service. In education, the low-level of negative emotions are "early warning signals" about student confidence in a diminished university. At a higher level, negative emotions can lead to a variety of coping behaviors such as complaint, sharing a negative word-of-mouth, and switching intention. The study employed the Partial Least Square - Structural Equation Modeling to analyze the relationship between negative emotions and switching decisions in a sample of 374 students who are studying at the University of Labour and Social Affairs, Ho Chi Minh campus (ULSA2). The empirical results revealed that there is a positive impact of anger, frustration on the complaint, and negative word-of-mouth. The regret leads to switching intention. Result of the research are references to university managers in policy planning.
\end{abstract}

Keywords: Negative emotion; Coping behavior; Student; Switching Decision; University.

\footnotetext{
${ }^{*}$ Corresponding author.

E-mail address: ngocbh.16ae@ou.edu.vn
}

https://doi.org/10.25073/2588-1159/vnuer.4361 


\title{
Tác động của cảm xúc tiêu cực đến quyết định chuyển đổi cơ sở học tập của sinh viên
}

\author{
Bùi Hoàng Ngọc* \\ Khoa Sau Đại học, Truò̀ng Đại học Mở Thành phố Hồ Chí Minh, \\ 97 Võ Văn Tần, Phuờng 6, Quận 3, Thành phố Hồ Chí Minh, Việt Nam \\ Nhận ngày 02 tháng 02 năm 2020 \\ Chỉnh sửa ngày 06 tháng 4 năm 2020; Chấp nhận đăng ngày 08 tháng 4 năm 2020
}

\begin{abstract}
Tóm tắt: Cảm xúc tiêu cực là kết quả của những trải nghiệm không hài lòng khi sử dụng sản phẩm hay dịch vụ. Trong lĩnh vực giáo dục đại học, cảm xúc tiêu cực ở mức độ thấp là "tín hiệu cảnh báo sóm" về niềm tin của sinh viên đối với cơ sở đào tạo bị giảm sút. Ở mức độ nghiêm trọng, cảm xúc tiêu cực có thể dẫn đến nhiều hành vi đối phó khác nhau như: Than phiền, chia sẻ trải nghiệm tiêu cực, hình thành ý định chuyển đổi rồi chuyển thành quyết định chuyển đổi chính thức. Nghiên cứu này sử dụng mô hình cấu trúc tuyến tính bình phương từng phần nhỏ nhất (PLS-SEM) cho mẫu nghiên cứu gồm 374 sinh viên. Kết quả thực nghiệm cho thấy tức giận, chán nản tác động mạnh tới than phiền và truyền miệng tiêu cực, trong khi hối tiếc sẽ hình thành trực tiếp ý định chuyển đổi cơ sở học tập. Kết quả nghiên cứu là tài liệu tham khảo cho các nhà quản lý ở Trường Đại học trong việc hoạch định chính sách.
\end{abstract}

Tù khóa: Cảm xúc tiêu cực, Hành vi đối phó, Sinh viên, Quyết định chuyển đổi, Trường Đại học.

\section{Giới thiệu}

Cảm xúc tiêu cực được nghiên cứu rộng rãi trong lĩnh vực marketing, nhưng còn khá khiêm tốn trong lĩnh vực giáo dục. Việc chuyển đổi tư tưởng giáo dục từ lấy giáo viên/giảng viên làm trung tâm sang phương thức lấy người học làm trung tâm đã thay đổi căn bản nhận thức về tầm quan trọng của yếu tố cảm xúc người học, nâng "tính dịch vụ" trong đào tạo của các Trường Đại học. Khác với các ngành sản xuất, có thể nói ngành dịch vụ nói chung và giáo dục đại học nói riêng cung cấp các dịch vụ vô hình, mang tính phức tạp cao, khó ổn định và không thể tách rời với chính thương hiệu của cơ sở đào tạo $[1,2]$. Theo các lý thuyết về hành vi của người tiêu dùng/khách hàng thì lỗi/thất bại trong việc cung cấp dịch vụ là không thể tránh

\footnotetext{
* Tác giả liên hệ.

Địa chi email: ngocbh.16ae@ou.edu.vn

https://doi.org/10.25073/2588-1159/vnuer.4361
}

khỏi, điều này dẫn đến những cảm xúc tiêu cực của khách hàng/người học.

Ở góc độ của sinh viên, dấu hiệu dễ nhận biết nhất và mức độ nhẹ của cảm xúc tiêu cực là kết quả học tập bị giảm sút [3], đầu tư ít thời gian cho việc học tập [4], cam chịu không làm bài thay vì cố gắng tìm kiếm các giải pháp khác [5], tự ý phá vỡ các quy định học tập của bản thân/nhà trường [6]. Về nguyên nhân, tổng hợp các theo nghiên cứu trước đây cho thấy cảm xúc tiêu cực của sinh viên bắt nguồn từ: Sự chưa ổn định về tâm lý, chất lượng cơ sở vật chất của cơ sở đào tạo, chất lượng đội ngũ giảng viên, chương trình đào tạo, áp lực thành tích học tập từ bản thân/gia đình/nhà trường. Ở mức độ nặng, cảm xúc tiêu cực thường gây ra các phản ứng cực đoan như: lo lắng [7]; cô đơn [8]; tuyệt vọng [9]; trầm cảm [10]; thậm chí có thể dẫn đến ý định tự tử [11].

Ở góc độ của cơ sở đào tạo, khi sinh viên có cảm xúc tiêu cực sẽ khuyến khích họ hình thành các hành vi đối phó khác nhau nhằm giải tỏa áp lực và gia tăng cảm xúc thoải mái cho bản thân. 
Ba hành vi/chiến lược đối phó tiêu biểu của sinh viên được tìm thấy trong các nghiên cứu thực nghiệm là: Than phiền, chia sẻ trải nghiệm tiêu cực và ý định chuyển đổi. Cả ba hành vi đối phó này đều làm giảm sút niềm tin của sinh viên, ảnh hưởng trực tiếp tới công tác tuyển sinh. Thậm chí nếu xuất hiện nhiều ý định chuyển đổi thì đây được xem là hành vi nghiêm trọng nhất vì nó ảnh hưởng tới hình ảnh và danh tiếng của cơ sở đào tạo đó trong cộng đồng.

Theo thuyết hành vi dự định thì trước khi ra được quyết định chuyển đổi (hành vi thực sự) các cá nhân phải hình thành ý định chuyển đổi (xu hướng hành vi) [12]. Ý định chuyển đổi lại được cấu thành từ ba thành tố: i) Thái độ; ii) Chuẩn chủ quan; iii) Nhận thức kiểm soát hành vi. Các nghiên cứu thực nghiệm trước đây như nghiên cứu [3, 4] đã chỉ ra mối liên hệ giữa cảm xúc tiêu cực và kết quả học tập của sinh viên, nhưng các nghiên cứu này nghiên cứu riêng lẻ từng loại cảm xúc tiêu cực (lo lắng, tức giận, chán nản, xấu hổ) hoặc từng loại hành vi đối phó (than phiền, chia sẻ trải nghiệm tiêu cực). Việc phân tích sâu từng loại cảm xúc tiêu cực giúp đề xuất các hàm ý quản trị, nhưng lại cung cấp cái nhìn thiếu tính tổng quát. Hơn nữa, các nghiên cứu trước chưa đề cập đển một hành vi

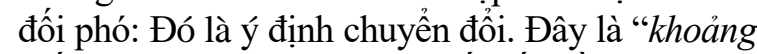
trống” mà nghiên cứu này muốn lấp đầy.

\section{Cơ sở lý thuyết và lược khảo các nghiên cứu trước}

\subsection{Lý thuyết nền}

Theo tâm lý học thì cảm xúc là một trạng thái tâm lý có "tính nguyên thủy" của con người. Để hình thành cảm xúc thì cần có đối tượng/sự kiện bên ngoài tác động. Như vậy cảm xúc có thể hiểu là "sự phản ưng/rung động bên trong của con người trước tác động của ngoại cảnh". Xét ở góc độ marketing thì cảm xúc của khách hàng là kết quả của những trải nghiệm khi sử dụng sản phẩm hay dịch vụ [13]. Nói cách khác, để hình thành nên cảm xúc thì khách hàng luôn có sự so sánh thực tế trải nghiệm với kỳ vọng của chính họ trước khi trải nghiệm, tức là họ phải trải qua quá trình đánh giá. "Đánh giá là một quá trình khi cá nhân nhận thức một sự việc cu thể có liên quan đến sự hạnh phúc của anh ta/cô ta hay không, và nếu có thì theo cách nào" [14]. Một sự việc xảy ra nếu gây ảnh hưởng tiêu cực đến nguồn lực cá nhân hoặc gây nguy hại đến hạnh phúc đều dẫn đến sự căng thẳng tâm lý $[2,15]$, nếu ảnh hưởng tích cực thì gây hứng thú và tạo động lực [16].

Xét theo thuyết hành vi dự định thì lý thuyết đánh giá nhận thức mới hình thành nên các xu hướng hành vi. Để biến các xu hướng hành vi này thành hành vi thực sự các cá nhân phải thông qua một quá trình nữa đó là quá trình nhận thức kiểm soát hành vi. Tức là các cá nhân sẽ phải tham khảo thêm ý kiến của những người có liên quan, đồng thời dự đoán những kết quả có thể xảy ra của việc hành động hay việc không hành động.

Ứng dụng hai lý thuyết này vào mối liên hệ giữa cảm xúc tiêu cực và quyết định chuyển đổi cơ sở học tập của sinh viên thu hút được sự chú ý của cả sinh viên, giảng viên và các nhà quản lý giáo dục. Sự hấp dẫn của chủ đề nằm ở chỗ cùng một sự việc cụ thể có thể hình thành nhiều cảm xúc tiêu cực khác nhau, và khi gặp cảm xúc tiêu cực sinh viên có nhiều hành vi đối phó khác nhau. Các khía cạnh này sẽ được phân tích ở phần lược khảo các nghiên cứu trước.

\subsection{Lược khảo các nghiên cứu trước}

Cảm xúc học tập được khởi nguồn từ nghiên cứu [17] nhưng nghiên cứu có tính tổng hợp là nghiên cứu [18]. Theo đó có nhiều dạng cảm xúc tiêu cực của sinh viên được tìm thây đó là: tức giận, lo lắng, chán nản, cô đơn, trầm cảm, tuyệt vọng, xấu hồ, và ý định tự tử. Để dễ phân biệt, có thể tạm phân cảm xúc tiêu cực thành các mức độ: Tức giận, chán nản và hối tiếc [18].

Cảm xúc là sự phản ứng bên trong con người với các tác động từ ngoại cảnh, do vậy các cảm xúc tiêu cực khác nhau của sinh viên là do sinh viên quy đối tượng chịu trách nhiệm gây ra lỗi khác nhau, cụ thể: Giận dữ có khuynh hướng xuất hiện khi cá nhân cho rằng sự việc gây căng thẳng xảy ra là do lỗi của đối tượng cụ thể bên ngoài (ví dụ: Chất lượng phòng học, trang thiết bị phục vụ giảng dạy, các tiện ích hỗ 
trợ/bổ sung cho hoạt động đào tạo như thư viện, phòng thí nghiệm, kí túc xá, căn tin, hoạt động thể dục thể thao,...); Chán nản là do cá nhân quy trách nhiệm cho những yếu tố tình huống không thể kiểm soát (ví dụ: Sự thay đổi chương trình đào tạo, chuẩn đầu ra, hay các hoạt động kiến tập thực tập, tư vấn nghề nghiệp không được thực hiện liên tục hoặc thiếu tính thực tế,...); Hối tiếc là suy nghĩ cho rằng lỗi đó là do bản thân, tức là cảm xúc tiêu cực mà họ nhận phải hôm nay là do sự lựa chọn/quyết định của chính họ trước đây (ví dụ: tình trạng của tôi bây giờ sẽ khác đi nếu trước đây tôi chọn cơ sở học tập khác, hoặc tôi lựa chọn một ngành học khác,...).

Đối phó là quá trình các cá nhân kiểm soát những nhu cầu (bên trong hoặc bên ngoài) được hình thành từ mối quan hệ căng thẳng giữa cá nhân - môi trường - cơ chế và những cảm xúc mà nhu cầu này tạo ra $[19,20]$. Sự thất bại trong việc cung cấp dịch vụ đào tạo được xem là một tình huống gây nên sự căng thẳng trong mối quan hệ giữa sinh viên - cơ sở giáo dục chương trình đào tạo [6]. Vì vậy, chiến lược đối phó đối với sự thất bại của dịch vụ đào tạo là những cố gắng về nhận thức và tình cảm của sinh viên nhằm kiểm soát nhu cầu của bản thân trong những tình huống này $[21,22]$.

Có hai loại chiến lược đối phó là: (1) Đối phó tập trung vào vấn đề hay hướng đến việc kiểm soát và thay đổi vấn đề gây nên sự căng thẳng; và (2) Đối phó tập trung vào tình cảm hay hướng đến việc điều chỉnh phản ứng cảm xúc đối với vấn đề [14]. Chiến lược đối phó thứ ba là né tránh [23], hàm ý việc cá nhân rời bỏ tình huống gây căng thẳng và chuyển sang sử dụng dịch vụ của nhà cung cấp khác $[24,25]$. Úng dụng vào lĩnh vực giáo dục đại học, cho dù sinh viên lựa chọn chiến lược đối phó nào, họ cũng cần biết ai là người chịu trách nhiệm cho các tình huống căng thẳng đó và khi biết ai phải chịu trách nhiệm, hành vi đối phó sẽ hướng đến đối tượng đó [3]. Đơn cử, sinh viên sẽ sử dụng chiến lược tập trung vào vấn đề (ví dụ: Than phiền) khi xác định người chịu trách nhiệm là các bên thứ ba (giảng viên, chuyên viên giải quyết các thủ tục hành chính) [7]. Trái ngược với cách đối phó tập trung vào vấn đề, chiến lược đối phó tập trung vào tình cảm (như chia sẻ trải nghiệm buồn) thường đổ lỗi cho chính bản thân mình, và do đó sinh viên sẽ chia sẻ trải nghiệm thất vọng này với gia đình, bạn bè hay người quen biết để xua tan cảm giác bực mình hay chán nản [8]. Cuối cùng, đối với cách đối phó tập trung vào việc né tránh, tức là sinh viên có thể lựa chọn im lặng rút lui và hình thành ý định chuyển sang một cơ sở học tập khác do cho rằng việc cố gắng giải quyết tình huống sẽ chỉ làm họ tốn kém thời gian và nỗ lực [4].

\subsection{Mối liên kết giũ̃a cảm xúc tiêu cực và hành vi đối phó}

Theo lý thuyết đánh giá nhận thức thì mối liên kết giữa những cảm xúc cụ thể và chiến lược đối phó rất phức tạp [14]. Vì vậy, để có thể hiểu sâu hơn hành vi đối phó của sinh viên cần có sự hiểu biết toàn diện về kỳ vọng của sinh viên trước khi nhập học và phản ứng cảm xúc của sinh viên trong bối cảnh những kỳ vọng đó không đạt được. Cụ thể hơn, nghiên cứu này muốn chỉ ra được mối liên kêt giữa ba loại cảm xúc tiêu cực gồm: Giận dữ, chán nản và hối tiếc với ba loại hành vi đối phó là: Than phiền, chia sẻ trải nghiệm tiêu cực và ý định chuyển đổi. Từ đó đề xuất được khung lý thuyết cho cơ chế truyền dẫn từ cảm xúc tiêu cực đến quyết định chuyển đổi cơ sở học tập của sinh viên.

Giận dữ là cảm xúc có tính hồi tưởng và có khuynh hướng xuất hiện khi cá nhân gán ghép một sự việc không phù hợp với mục tiêu bản thân cho một đối tượng bên ngoài (ví dụ: Cơ sở vật chất, chất lượng đội ngũ giảng viên). Giận dữ là cảm xúc có tính thời điểm và là phản ứng thường thấy đối với những trải nghiệm học tập không như kỳ vọng của sinh viên do lỗi của cơ sở đào tạo $[6,26]$. Các nghiên cứu trước đây khẳng định rằng giận dữ làm giảm động lực học tập và thành tích của sinh viên, đồng thời thúc đầy các hành vi trả đũa $[3,18,21]$. Sự giận dữ thúc đẩy sinh viên than phiền và chia sẻ trải nghiệm tiêu cực $[6,18]$; còn sự phân biệt đối xử và cảm xúc tiêu cực có "tính lũy kế” [22]. Tức là, sự phân biệt đối xử trong đào tạo ở hiện tại (ví dụ như sự phân biệt trong quốc tịch, màu da, địa vị xã hội của cha mẹ) và cảm xúc tiêu cực là cả một quá trình tích lũy lâu dài trước đó. Ngoài 
ra kết quả học tập của các học kỳ trước đó cũng có ảnh hưởng đển cảm xúc tiêu cực ở kỳ hiện tại. Vì vậy, nghiên cứu này đề xuất các giả thuyết sau: phiền

$\mathrm{H}_{1 \mathrm{a}}$ : Giận dữ có tác động tích cực đến than

$\mathrm{H}_{1 \mathrm{~b}}$ : Giận dữ có tác động cùng chiều với chia sẻ trải nghiệm tiêu cực

Chán nản cũng là cảm xúc hồi tưởng. Nhưng khác với giận dữ, chán nản có khuynh hướng xuất hiện khi sinh viên gán ghép một sự kiện không đúng với kỳ vọng của bản thân cho các nhân tố tình huống [21]. Nói cách khác, sinh viên cho rằng các tình huống không kiểm soát được (ví dụ: Thay đổi chuẩn đầu ra, thay đổi chương trình đào tạo) là nguyên nhân của các sự việc gây căng thẳng. Do đó, chán nản là một cảm xúc khác biệt với giận dữ [25]. Lý thuyết đánh giá nhận thức khẳng định chán nản là bước đầu tiên thúc đẩy người tiêu dùng/sinh viên có những hành vi đối phó thích hợp nhằm loại bỏ cảm giác khó chịu này [14]. Tương tự giận dữ, sinh viên khi có cảm giác chán nản sẽ có ý định than phiền hoặc chia sẻ trải nghiệm tiêu cực với người khác vì những hành vi này sẽ giúp họ cảm thấy thoải mái hơn [20, 21, 26]. Vì vậy, nghiên cứu này đề xuất các giả thuyết sau:

$\mathrm{H}_{2 \mathrm{a}}$ : Chán nản có mối liên hệ cùng chiều với than phiền

$\mathrm{H}_{2 b}$ : Chán nản có mối liên hệ cùng chiều với chia sẻ trải nghiệm tiêu cực

Hối tiếc có khuynh hướng xuất hiện khi cá nhân cho rằng sự việc gây căng thẳng xảy ra là do lỗi của bản thân [13]. Nói cách khác, hối tiếc thường hình thành khi cá nhân tưởng tượng rằng tình huống hiện tại có thể sẽ tốt hơn nếu trước đây bản thân có hành động khác đi. Khác với các lĩnh vực dịch vụ khác, sự hối tiếc trong lĩnh vực giáo dục không thể kết thúc ngay mà nó "lớn dần" theo theo thời gian nếu các cảm xúc tiêu cực không được giải quyết triệt để. Tức là giận dữ và chán nản có thể xuất hiện ngay năm học thứ nhất, nhưng sự hối tiếc sẽ hình thành và "lớn dần" từ đầu năm học thứ hai. Vì cần thời gian và quy lỗi cho bản thân nên khi cảm xúc hối tiếc hình thành thì sinh viên thường tìm kiếm sự thông cảm từ người khác (bạn bè, gia đình). Vì vậy, nghiên cứu này đề xuất giả thuyết sau:

$\mathrm{H}_{3 \mathrm{a}}$ : Hối tiếc có mối liên hệ cùng chiều với ý định chuyển đổi

$\mathrm{H}_{3 \mathrm{~b}}$ : Hối tiếc có mối liên hệ cùng chiều với quyết định chuyển đổi

Nỗ lực hạn chế cảm xúc tiêu cực không chỉ phụ thuộc vào bản thân sinh viên mà còn phụ thuộc vào cơ sở đào tạo $[4,22]$. Thật không may là việc thay đổi từ phía cơ sở đào tạo luôn cần thời gian, sự đầu tư tài chính, thậm chí là trông chờ cả thay đổi trong hệ thống văn bản pháp luật. Trong rất nhiều trường hợp, sự giận dữ của sinh viên thường không cải thiện được tình hình mà để lại những hậu quả tiêu cực cho chính họ. Sinh viên có thể lựa chọn chiến lược né tránh, tức là họ lựa chọn giải pháp im lặng rút lui và hình thành ý định chuyển sang một cơ sở học tập khác [4]. Vì vậy, nghiên cứu này đề xuất các giả thuyết sau:

$\mathrm{H}_{4 \mathrm{a}}$ : Than phiền có tác động cùng chiều với ý định chuyển đổi

$\mathrm{H}_{4 b}$ : Chia sẻ trải nghiệm tiêu cực có tác động cùng chiều với ý định chuyển đổi

\subsection{Biến kiểm soát}

Theo lý thuyết hành vi dự định thì để biến một xu hướng hành vi thành hành vi thực sự, các cá nhân phải thông qua quá trình nhận thức kiểm soát hành vi. Tức là phải tham khảo thêm ý kiến/quan điểm của nhóm tư vấn (gia đình, bạn bè, thầy cô) và cân nhắc đến tất cả các kết quả có thể xảy ra/không xảy ra bởi việc ra quyết định đó. Theo đó đằng sau một quyết định luôn là một "chi phí co hội" cho quyết định đó. Việc sinh viên quyết định chuyển đổi sang sử dụng dịch vụ đào tạo của cơ sở đào tạo khác cũng không đơn giản, bên cạnh yếu tố thủ tục hành chính còn phải cân nhắc đến năng lực thực tế của sinh viên và chi phí cho việc chuyển đổi (gồm học phí và các loại phí đào tạo khác) của cả hai cơ sở. Nếu chi phí chuyển đổi sang cơ sở đào tạo mới tương đương hoặc thấp hơn cơ sở đào tạo cũ thì việc biến ý định chuyển đổi thành quyết định chuyển đổi sẽ ít để lại hậu quả tiêu cực hơn [22]. Trong cơ chế truyền dẫn từ cảm 
xúc tiêu cực đến quyết định chuyển đổi cơ sở học tập của sinh viên thì biến chi phí chuyển đổi đóng vai trò là biến kiểm soát. Do vậy, nghiên cứu này đề xuất các giả thuyết như sau:

$\mathrm{H}_{5}$ : Chi phí chuyển đổi có tác động cùng chiều với quyết định chuyển đổi

$\mathrm{H}_{6}$ : Ý định chuyển đổi có tác động cùng chiều với quyết định chuyển đổi

\section{Phương pháp nghiên cứu}

\subsection{Khái niệm và thang đo lường}

Các khái niệm và thang đo lường sử dụng trong nghiên cứu này được tác giả kế thừa từ các nghiên cứu $[3,6,13,18]$. Bảng hỏi được thiết kế dựa trên thang đo Likert 5 mức độ với: 1: Hoàn toàn không đồng ý; 3: Không có ý kiến; 5: Hoàn toàn đồng ý. Kết quả phân tích chi tiết các mục hỏi sẽ được trình bày trong phần 4.

\subsection{Dũ liệu và quá trình thu thập dĩ liệu}

Đối tượng khảo sát là sinh viên của Trường Đại học Lao động Xã hội (cơ sở thành phố Hồ Chí Minh). Do tác giả hiện là giảng viên của Trường Đại học Lao động Xã hội (cơ sở thành phố Hồ Chí Minh) nên việc thu thập dữ liệu được tác giả và 10 sinh viên năm 3 ngành quản trị kinh doanh đã được huấn luyện trực tiếp tiến hành. Những đáp viên được lựa chọn ngẫu nhiên trong số sinh viên của Trường, được yêu cầu hoàn thành bảng hỏi và gửi lại trực tiếp cho người điều tra ngay khi hoàn thành. 400 bảng hỏi đã được phát ra, nhóm nghiên cứu thu lại 374 bảng hỏi hợp lệ, đạt tỉ lệ 93,5\%.

\subsection{Phuơng pháp nghiên cúu}

Nghiên cứu sử dụng phần mềm kinh tế lượng để phân tích thống kê mô tả, việc kiểm định các giả thuyết nghiên cứu được thực hiện bằng mô hình cấu trúc tuyến tính bình phương riêng phần nhỏ nhất PLS-SEM do [27] giới thiệu. Hiện nay, mô hình SEM được sử dụng phổ biến trong lĩnh vực phân tích hành vi.

\section{Kết quả nghiên cứu và thảo luận}

\subsection{Thống kê mô tả}

Trường Đại học Lao động Xã hội (cơ sở Thành phố Hồ Chí Minh) hiện đào tạo các chuyên ngành chính gồm: Bảo hiểm, Kế toán, Quản trị kinh doanh, Quản trị nguồn nhân lực, Luật kinh tế và Công tác xã hội. Mẫu khảo sát thu thập từ 374 sinh viên được phân tích thống kê mô tả như ở bảng 1 . Theo đó, sinh viên năm 1 chiếm tỉ lệ $29,9 \%$, năm 2 là $25,1 \%$, năm 3 là $42,8 \%$. Số lượng sinh viên năm 4 bị hạn chế do thời gian khảo sát trùng với thời gian đi thực tập cuối khóa. Phân theo ngành học, ngành bảo hiểm chiếm $8,02 \%$, ngành quản trị kinh doanh chiếm $33,16 \%$, ngành quản trị nguồn nhân lực là $24,06 \%$, số còn lại là các ngành học khác.

Bảng 1. Thống kê mô tả mẫu nghiên cứu

\begin{tabular}{|l|l|l|l|l|l|}
\hline \multirow{2}{*}{ Ngành học } & $\begin{array}{l}\text { Giới } \\
\text { tính }\end{array}$ & $\begin{array}{l}\text { Năm } \\
1\end{array}$ & $\begin{array}{l}\text { Năm } \\
2\end{array}$ & $\begin{array}{l}\text { Năm } \\
3\end{array}$ & $\begin{array}{l}\text { Năm } \\
4\end{array}$ \\
\hline \multirow{2}{*}{$\begin{array}{l}\text { Ngành Bảo } \\
\text { hiểm }\end{array}$} & Nam & 2 & 3 & 2 & 0 \\
\cline { 2 - 6 } & Nữ & 6 & 12 & 5 & 0 \\
\hline $\begin{array}{l}\text { Ngành Quản } \\
\text { trị kinh } \\
\text { doanh }\end{array}$ & Nam & $24 *$ & 5 & 25 & 5 \\
\cline { 2 - 6 } & Nữ & $25 *$ & $16 *$ & 21 & 3 \\
\hline $\begin{array}{l}\text { Ngành Luật } \\
\text { kinh tế }\end{array}$ & Nam & 5 & 2 & 0 & 0 \\
\cline { 2 - 6 } & Nữ & 9 & 15 & 0 & 0 \\
\hline $\begin{array}{l}\text { Ngành Kế } \\
\text { toán }\end{array}$ & Nam & 2 & 1 & 3 & 0 \\
\cline { 2 - 6 } $\begin{array}{l}\text { Nữnh Quản } \\
\text { trị nguồn } \\
\text { nhân lực }\end{array}$ & $23 *$ & $18 *$ & 26 & 0 \\
\cline { 2 - 6 } & Naũ & 3 & 3 & 33 & 0 \\
\hline $\begin{array}{l}\text { Ngành công } \\
\text { tác xã hội }\end{array}$ & Nam & 3 & 2 & 2 & 0 \\
\cline { 2 - 6 } & Nữ & 6 & 5 & 8 & 0 \\
\hline Tống cộng & & 112 & 94 & 160 & 8 \\
\hline
\end{tabular}

Ghi chú: Dấu * Minh họa cho trong nhóm có sinh viên đã có quyết định nghỉ học hoặc bảo lưu.

\subsection{Kiểm định thang đo}

Để một thang đo có độ tin cậy thì nó phải đạt được đồng thời hai tiêu chí là: Độ giá trị hội tụ và độ giá trị phân biệt [27]. Do vậy, trước tiên bài viết đi kiểm định độ giá trị hội tụ của các thang đo thông qua các hệ số Cronbach's Alpha (kí hiệu là $C A$ ), hệ số tải nhân tố, độ tin cậy tổng hợp (kí hiệu là $C R$ ) và phương sai trích (ki hiệu là $A V E$ ). 
Bảng 2. Kiểm định hệ số Cronbach's Alpla, $\mathrm{CR}$ và $\mathrm{AVE}$

\begin{tabular}{|c|c|c|c|c|}
\hline Mục hỏi & Hệ số tải nhân tố & $\mathrm{CA}$ & $\mathrm{CR}$ & AVE \\
\hline $\begin{array}{l}\text { Thang đo tức giận: Cơ sở đào tạo không đáp ứng được mong muốn } \\
\text { của tôi, nên,... }\end{array}$ & & 0,829 & 0,880 & 0,595 \\
\hline ... tôi cảm thấy không thoải mái & 0,751 & & & \\
\hline ... tôi cảm thấy ấm ức & 0,818 & & & \\
\hline ... tôi cảm thấy bực mình & 0,786 & & & \\
\hline ... tôi cảm thấy giận dữ & 0,783 & & & \\
\hline$\ldots$ tôi cảm thấy thù ghét & 0,714 & & & \\
\hline $\begin{array}{l}\text { Thang đo chán nản: Cơ sở đào tạo không đáp ứng được mong } \\
\text { muốn của tôi, nên,... }\end{array}$ & & 0,767 & 0,866 & 0,683 \\
\hline ... tôi cảm thấy chán nản & 0,779 & & & \\
\hline ... tôi cảm thấy cam chịu & 0,820 & & & \\
\hline ... tôi cảm thấy bất lực & 0,877 & & & \\
\hline Thang đo hối tiếc: & & 0,683 & 0,863 & 0,758 \\
\hline Tôi cảm thấy hối tiếc vì đã chọn cơ sở học tập này & 0,890 & & & \\
\hline Nếu được chọn lại, tôi sẽ chọn cơ sở học tập khác & $(*)$ & & & \\
\hline $\begin{array}{l}\text { Hoàn cảnh bây giờ của tôi có thể tốt hơn, nếu trước đây tôi chọn cơ } \\
\text { sở học tập khác }\end{array}$ & 0,851 & & & \\
\hline Thang đo than phiền: & & 0,886 & 0,916 & 0,687 \\
\hline Tôi phản ánh sự thất vọng với cố vấn học tập & 0,814 & & & \\
\hline Tôi trình bày sự thất vọng với lãnh đạo khoa và phòng ban chuyên trách & 0,818 & & & \\
\hline Tôi thảo luận với với cố vấn học tập để tìm kiếm giải pháp & 0,865 & & & \\
\hline $\begin{array}{l}\text { Tôi trình bày cách tôi bị đối xử với bộ phận chuyên trách quản lý } \\
\text { sinh viên }\end{array}$ & 0,841 & & & \\
\hline Tôi sẽ có ý với lãnh đạo Nhà trường tại các buổi đối thoại với sinh viên & 0,804 & & & \\
\hline Thang đo truyền miệng tiêu cực: & & 0,757 & 0,861 & 0,674 \\
\hline Tôi kể với bạn bè tôi về vấn đề tôi gặp phải & 0,843 & & & \\
\hline Tôi kể với gia đình về vấn đề tôi gặp phải & 0,832 & & & \\
\hline Tôi khuyên người khác không nên lựa chọn cơ sở đào tạo này & 0,786 & & & \\
\hline Thang đo ý định chuyển đổi: & & 0,808 & 0,874 & 0,636 \\
\hline Tôi không thích cơ sở học tập này nữa & 0,771 & & & \\
\hline Tôi sẽ không học cơ sở đào tạo này ở những trình độ cao hơn & 0,825 & & & \\
\hline Tôi tìm hiểu chất lượng học tập ở các cơ sở đào tạo khác & 0,862 & & & \\
\hline Tôi có ý định chuyển đổi cơ sở học tập & 0,725 & & & \\
\hline Thang đo khả năng chuyển đổi: & & 0,781 & 0,857 & 0,601 \\
\hline Cơ sở học tập khác có học phí phù hợp với khả năng chi trả của tôi & 0,799 & & & \\
\hline Cơ sở học tập khác có hoạt động ngoại khóa phù hợp & 0,740 & & & \\
\hline Cơ sở học tập khác có vị trí thuận tiện cho việc di chuyển & 0,735 & & & \\
\hline Cơ sở học tập khác có thương hiệu về đào tạo & 0,821 & & & \\
\hline Thang đo quyết định chuyển đổi: & & 0,904 & 0,940 & 0,840 \\
\hline Gia đình ủng hộ tôi chuyển đổi & 0,910 & & & \\
\hline Bạn bè ủng hộ tôi chuyển đổi & 0,929 & & & \\
\hline Tôi đã chọn được thời điểm chuyển đổi & 0,909 & & & \\
\hline
\end{tabular}

Ghi chú: (*) là mục hỏi không đạt yêu cầu của kiểm định. 
Bảng 3. Kiểm định độ phân biệt bằng chỉ số Heterotrait-Monotrait Ratio (HTMT)

\begin{tabular}{|l|l|l|l|l|l|l|l|l|}
\hline & $(1)$ & $(2)$ & $(3)$ & $(4)$ & $(5)$ & $(6)$ & $(7)$ & $(8)$ \\
\hline$(1)$ & 0,771 & & & & & & & \\
\hline$(2)$ & 0,540 & 0,829 & & & & & & \\
\hline$(3)$ & 0,555 & 0,739 & 0,775 & & & & & \\
\hline$(4)$ & 0,661 & 0,566 & 0,513 & 0,826 & & & & \\
\hline$(5)$ & 0,611 & 0,379 & 0,373 & 0,544 & 0,871 & & & \\
\hline$(6)$ & 0,668 & 0,472 & 0,434 & 0,587 & 0,670 & 0,916 & & \\
\hline$(7)$ & 0,590 & 0,588 & 0,525 & 0,579 & 0,449 & 0,491 & 0,797 & \\
\hline$(8)$ & 0,625 & 0,654 & 0,538 & 0,576 & 0,471 & 0,603 & 0,549 & 0,821 \\
\hline
\end{tabular}

Ghi chú: (1) Tức giận; (2) Than phiền; (3) Khả năng chuyển đổi; (4) Chán nản; (5) Hối tiếc;

(6) Quyết định chuyển đổi; (7) Ý định chuyển đổi; (8) Truyền miệng tiêu cực.

Kết quả kiểm định ở bảng 2 cho thấy tất cả các hệ số tải nhân tố đều $>0,7$, hệ số Cronbach's Alpha đều > 0,6, hệ số độ tin cậy tổng hợp đều $>0,8$ và hệ số phương sai trích đều $>0,5$. Như vậy là các thang đo của nghiên cứu đều thỏa mãn độ giá trị hội tụ [28]. Kế tiếp, bài viết kiểm tra độ giá trị phân biệt của các thang đo bằng cách sử dụng chỉ số HeterotraitMonotrait Ratio (HTMT). Kết quả kiểm định trong Bảng 3 cũng cho thấy tất cả các giá trị của các hệ số HTMT trên đường chéo chính đều lớn hơn giá trị cùng dòng, kết quả như vậy chứng tỏ thang đo đạt được độ giá trị phân biệt [29].

\section{Kiểm định giả thuyết}

Bước thứ ba, bài viết dựa theo quy trình được đề xuất bởi [27] để kiểm định các giả thuyết nghiên cứu đã đặt ra ở phần 2 bằng phương pháp ước lượng tuyến tính riêng phần nhỏ nhất PLS-SEM. Kết quả phân tích đường dẫn được minh họa trong Hình 1 và Bảng 4 dưới đây:

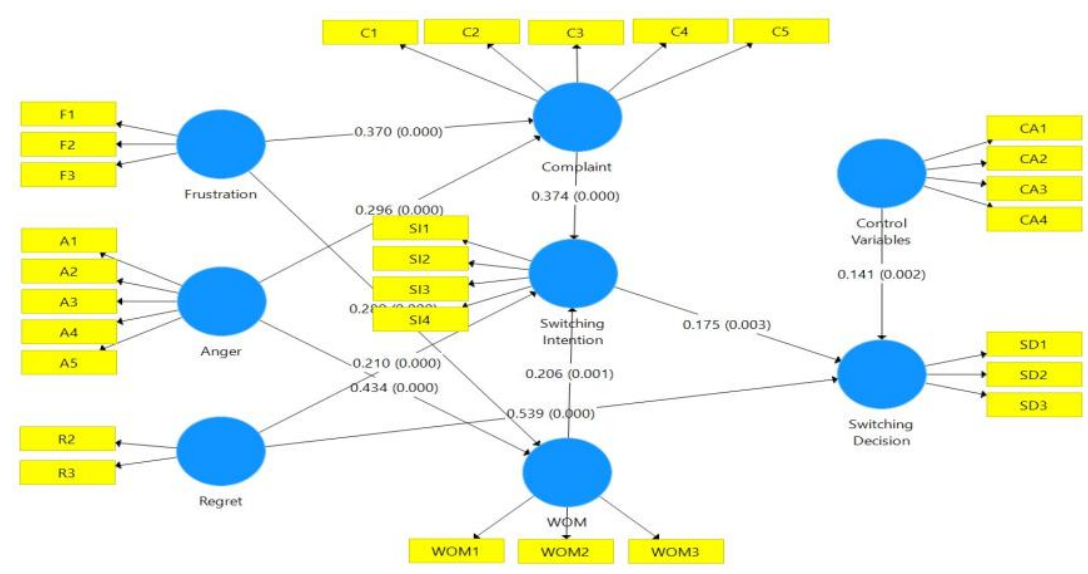

Hình 1. Kết quả phân tích đường dẫn.

Ghi chú: Frustration là thang đo chán nản, Anger là tức giận, Regret là hối tiếc, Complaint là than phiền, WOM là truyền miệng tiêu cực, Switching Intention là ý định chuyển đổi, Control variable là khả năng chuyển đồi, Swithching Decision là quyết định chuyển đổi. 
Bảng 4. Kết quả phân tích đường dẫn

\begin{tabular}{|c|c|c|c|c|c|}
\hline Các mối quan hệ & Giả thuyết & Hệ số $\beta$ & Sai số & t-value & Kết luận \\
\hline Tức giận $\rightarrow$ Than phiền & $\mathrm{H}_{1 \mathrm{a}}$ & 0,296 & 0,064 & $4,589 * * *$ & Ủng hộ \\
\hline Tức giận $\rightarrow$ Truyền miệng & $\mathrm{H}_{1 \mathrm{~b}}$ & 0,434 & 0,052 & $8,329 * * *$ & Ủng hộ \\
\hline Than phiền $\rightarrow$ Ý định chuyển đổi & $\mathrm{H}_{4 \mathrm{a}}$ & 0,374 & 0,065 & $5,725 * * *$ & Ủng hộ \\
\hline $\begin{array}{l}\text { Khả năng chuyển đổi } \rightarrow \text { Quyết định } \\
\text { chuyển đổi }\end{array}$ & $\mathrm{H}_{5}$ & 0,141 & 0,046 & $3,073 * *$ & Ủng hộ \\
\hline Chán nản $\rightarrow$ Than phiền & $\mathrm{H}_{2 \mathrm{a}}$ & 0,370 & 0,066 & $5,576 * * *$ & Ủng hộ \\
\hline Chán nản $\rightarrow$ Truyền miệng & $\mathrm{H}_{2 \mathrm{~b}}$ & 0,289 & 0,055 & $5,282 * * *$ & Ủng hộ \\
\hline Hối tiếc $\rightarrow$ Quyết định chuyển đổi & $\mathrm{H}_{3 \mathrm{~b}}$ & 0,539 & 0,045 & $11,94 * * *$ & Ủng hộ \\
\hline Hối tiếc $\rightarrow$ Ý định chuyển đổi & $\mathrm{H}_{3 \mathrm{a}}$ & 0,210 & 0,050 & $4,184 * * *$ & Ủng hộ \\
\hline $\begin{array}{l}\text { Ý định chuyển đổi } \rightarrow \text { Quyết định } \\
\text { chuyển đổi }\end{array}$ & $\mathrm{H}_{6}$ & 0,175 & 0,058 & $3,017 * *$ & Ủng hộ \\
\hline Truyền miệng $\rightarrow$ Ý định chuyển đổi & $\mathrm{H}_{4 \mathrm{~b}}$ & 0,206 & 0,063 & $3,255^{* *}$ & Ủng hộ \\
\hline Giá trị $\mathrm{R}^{2}$ & \multicolumn{5}{|c|}{$\begin{array}{l}\mathrm{R}^{2}(\text { Than phiền })=0,369 ;(\text { Truyền miệng })=0,438 ;(Y ́ \text { định } \\
\text { chuyển đồi) }=0,427 ; \text { (Quyết định chuyển đồi) }=0,508\end{array}$} \\
\hline Mức độ phù hợp của mô hình & \multicolumn{5}{|c|}{ Hệ số phù hợp tổng thể của mô hình = 0,918 } \\
\hline Độ lớn của tác động (Giá trị f²) & \multicolumn{5}{|c|}{$\begin{array}{l}\mathrm{f}^{2}(\text { Tức giận } \rightarrow \text { Than phiền })=0,078 ;(\text { Tức giận } \rightarrow \text { Truyền } \\
\text { miệng })=0,189\end{array}$} \\
\hline & \multicolumn{5}{|c|}{$\begin{array}{l}\mathrm{f}^{2}(\text { Than phiền } \rightarrow \text { Ý định chuyển đổi })=0,139 ;(\text { Khả năng } \\
\text { chuyển đổi } \rightarrow \text { Quyết định chuyển đổi) }=0,028 ;(\text { Chán nản } \\
\rightarrow \text { Than phiền })=0,123 ; \text { (Chán nản } \rightarrow \text { Truyền miệng) }= \\
0,084 \text {. (Hối tiếc } \rightarrow \text { Quyết định chuyển đổi) }=0,455 ; \text { (Hối } \\
\text { tiếc } \rightarrow \text { Ý định chuyển đồi) }=0,060 ;(\text { Ý định chuyển đồi } \rightarrow \\
\text { Quyết định chuyển đổi) }=0,040 ; \text { (Truyền miệng } \rightarrow \text { Ý định } \\
\text { chuyển đổi) }=0,038\end{array}$} \\
\hline$Q^{2}$ value & \multicolumn{5}{|c|}{$\begin{array}{l}\mathrm{Q}^{2}(\text { Than phiền })=0,210 ; \mathrm{Q}^{2}(\text { Truyền miệng tiêu cực })= \\
0,220 ; \mathrm{Q}^{2}(Y \text { Yịnh chuyển đồi })=0,249 ; \mathrm{Q}^{2}(\mathrm{Q} \text { uyết định } \\
\text { chuyển đồi })=0,427\end{array}$} \\
\hline
\end{tabular}

Ghi chú: *,**, *** là mức ý nghĩa tương ứng với $5 \%, 1 \%$, và $0,1 \%$.

Theo kết quả trong Bảng 4, thì tức giận đều gây ra hành vi đối phó là than phiền $(0,296)$ và truyền miệng tiêu cực $(0,434)$, hàm ý là khi sinh viên gặp các vấn đề về cảm xúc tiêu cực thì họ ưu tiên nhiều hơn cho việc chia sẻ các trải nghiệm này với người thân, bạn bè hơn là phản ảnh trực tiếp cho cố vấn học tập hoặc bộ phận phụ trách sinh viên. Ngược lại thì chán nản lại có tác động nhiều hơn đến hành vi than phiền. Đáng chú ý là sự hối tiếc tác động đồng thời đến cả sự hình thành ý định chuyển đổi và thúc đẩy quyết định chuyển đổi chính thức. Trong tất cả các hệ số tác động thì hệ số tác động của hối tiếc đến quyết định chuyển đổi là mạnh nhất $(=0,539)$, điều này cũng được khẳng định thông qua kiểm định độ lớn tác động $\left(\mathrm{f}^{2}=0,455\right)$. Hàm ý rằng, khi cảm xúc tiêu cực được đẩy tới mức hối tiếc thì sinh viên không cam chịu mà luôn tìm cho mình một hướng đi khác. Bằng chứng này rất có ý nghĩa đối với giảng viên và đặc biệt là các cán bộ quản lý giáo dục. Kết quả phân tích cũng chỉ ra mặc dù ý định chuyển đồi có tác động tích cực đến quyết định chuyển đổi $(\beta=0,175)$, tuy nhiên mức độ tác động này khá nhỏ $\left(\mathrm{f}^{2}=0,04\right)$, chứng tỏ sinh viên vần có sự dao động về mặt tâm lý hoặc do các thủ tục chuyển đổi (cả chiều đi và chiều đến) cũng chưa thực sự thuận lợi. Hệ số phù hợp tổng thể của mô hình $=0,918$, chứng tỏ mô hình nghiên cứu là phù hợp, hàm ý $91,8 \%$ sự biến động của 
quyết định chuyển đổi đã được giải thích bởi các biến mà mô hình đề xuất.

\section{Kết luận và hàm ý quản trị}

\subsection{Kết luận}

Nghiên cứu này được dựa trên lý thuyết về đánh giá nhận thức và lý thuyết hành vi dự định, cùng các nghiên cứu trước đây về mối quan hệ giữa cảm xúc tiêu cực và hành vi đối phó của khách hàng. Ứng dụng trong lĩnh vực giáo dục, với mẫu dữ liệu gồm 374 sinh viên đang học tập tại Trường Đại học Lao động Xã hội (cơ sở Thành phố Hồ Chí Minh) và bằng mô hình cấu trúc tuyến tính riêng phẩn nhỏ nhất PLS-SEM, kết quả thực nghiệm rút ra một số kết luận như sau:

Thứ nhất, Có mối quan hệ thuận chiều giữa cảm xúc tiêu cực và ý định chuyển đổi cơ sở học tập của sinh viên. Theo đó, cảm xúc tiêu cực càng nhiều thì ý định chuyển đổi càng lớn và ngược lại.

Thư hai, mặc dù khẳng định được ý định chuyển đổi sẽ dẫn tới quyết định chuyển đổi, nhưng mức độ tác động là chưa đủ độ lớn.

Kết luận của nghiên cứu này tương đồng với kết luận [18] cho 1.345 sinh viên ở Philippine, theo đó cảm xúc tiêu cực sẽ ảnh hưởng tiêu cực đến kết quả học tập của sinh viên và khuyến khích họ đưa ra các hành vi đối phó phù hợp. Hay nghiên cứu [26] cho 160 sinh viên tại bang Arizona và bang California (Mỹ). Điểm khác của nghiên cứu này là không dừng ở mức độ ảnh hưởng của cảm xúc tiêu cực đến kết quả học tập, mà mở rộng đến cả ý định chuyển đồi và quyết định chuyển đổi. Do vậy, nghiên cứu đã đóng góp nhất định cả giá trị học thuật và thực nghiệm cho các nghiên cứu tiếp theo về chủ đề cảm xúc tiêu cực của sinh viên.

\subsection{Hàm ý quản trị}

Nghiên cứu này tìm được bằng chứng là cảm xúc tiêu cực sẽ dẫn đến các hành vi đối phó gồm: than phiền, truyền miệng tiêu cực và ý định chuyển đổi. Sinh viên có nhiều lý do để than phiền trong suốt quá trình học tập như:
Than phiền về chất lượng của cơ sở vật chất, than phiền về chất lượng đào tạo, về học phí, về tiến độ học tập hay mức độ phức tạp của các môn học, v.v... [6]. Một điểm rất đáng lưu ý là việc than phiền có thể xuất phát từ chính sự chưa trưởng thành về tâm lý của sinh viên, chứ chưa hẳn là do cơ sở đào tạo [22]. Tuy nhiên, cơ sở đào tạo hay giảng viên không nên nhìn nhận hành vi than phiền của sinh viên theo chiều hướng tiêu cực, khi sinh viên than phiền là bản thân họ muốn nhận thấy những thay đổi tích cực. Điều này hàm ý sinh viên vẫn có niềm tin vào cơ sở đào tạo và mong muốn cơ sở đào tạo ngày càng trở lên chuyên nghiệp hơn [3].

Truyền miệng tiêu cực của sinh viên phải được xem là hành vi nghiêm trọng vì tính lây lan của nó. Để khắc phục hậu quả này, ngoài việc cải thiện các điều kiện học tập, chất lượng của đội ngũ giảng viên, sắp xếp hợp lý môn học, lịch học,,.. thì bộ phận quản lý sinh viên cần phải áp dụng cách giải thích hồi cứu [3]. Tức là nên giải thích về các biện pháp đã được đưa ra để hỗ trợ sinh viên, hoặc giải thích theo hướng đặt sinh viên vào vị trí của Trường Đại học để hiểu tại sao lại xảy ra sự cố như vậy. Tuy nhiên, cần tránh việc chối bỏ trách nhiệm của Trường Đại học hay đổ lỗi cho bên thứ ba [22].

Đối với Trường Đại học, ý định chuyển đổi của sinh viên là hành vi nghiêm trọng nhất vì nó ảnh hưởng trực tiếp tới công tác tuyển sinh, uy tín, hình ảnh, thương hiệu của Trường Đại học đó trong xã hội. Hiện nay, việc chuyển đổi giữa các Trường Đại học trong nước, hay giữa Trường Đại học trong nước với Trường Đại học nước ngoài đã có nhiều thuận lợi. Lưu ý rằng ý định chuyển đổi là một quá trình, tức là than phiền có thể xuất hiện nhiều ở sinh viên năm thứ nhất, còn chán nản cùng ý định chuyển đổi sẽ hình thành và "lón dần" từ sinh viên năm thứ hai [22]. Do vậy, các giải pháp để hạn chế sinh viên chuyển đồi cơ sở học tập cần được áp dụng đồng bộ, triệt để và mạnh mẽ ngay từ sinh viên năm nhất.

\subsection{Hạn chế và hướng nghiên cưu tiếp theo}

Bên cạnh kết quả thực nghiệm, nghiên cứu này cũng gặp một số hạn chế nhất định cho các 
nghiên cứu trong lĩnh vực hành vi. Thư nhất, Nghiên cứu được thực hiện cho đối tượng là sinh viên chính quy học tập theo hình thức học chế tín chỉ tập trung, mà chưa nghiên cứu các nhóm đối tượng khác như học viên cao học, sinh viên hệ đào tạo từ xa, sinh viên hệ vừa học vừa làm. Thứ hai, các biến số tính cách cá nhân như kết quả thi đầu vào đại học, địa vị xã hội của gia đình, hay tác động của các yếu tố đoàn thể (Đoàn thanh niên, Hội sinh viên) chưa được phân tích trong nghiên cứu này. Đây là hướng gợi mở cho các nghiên cứu tiếp theo về chủ đề cảm xúc tiêu cực của sinh viên.

\section{Tài liệu tham khảo}

[1] A.M. Susskind, Consumer Chán nản in the customer-server exchange: The role of attitudes toward complaining and information inadequacy related to service failures, Journal of Hospitality and Tourism Research 28(1) (2016) 21-43.

[2] J. Gao, D.L. Kerstetter, From sad to happy to happier: Emotion regulation strategies used during a vacation, Annals of Tourism Research 69 (2018) $1-14$.

[3] A.M.M. Garcia, The role of positive and negative emotions to academic performance, Undergraduate Thesis Proposal, San Beda College, 2016.

[4] A.C. Frenzel, R. Pekrun, T. Goetz, Perceived learning environment and students' emotional experiences: A multilevel analysis of mathematics classrooms, Learning and Instruction 17 (2007) 478-493.

[5] A.M. Isen, Positive affect, In T. Dalgleish, M. Power (Eds.), Handbook of cognition and emotion, New York: Wiley, 1999.

[6] R. Pekrun, T. Goetz, W. Titz, Academic Emotions in Students'Self-Regulated Learning and Achievement: A Program of Qualitative and Quantitative Research, Educational Psychologist 37 (2002) 91-106.

[7] O. Birgin, M. Baloglu, J. Catlioglu Hakan, R. Gurbuz Ramazan, Investigation of mathematics anxiety among sixth through eighth grade students in turkey, Learning and Individual Differences 20 (2010) 654-658.

[8] E.P. Galanaki, S.A. Polychronopoulou, T.K. Babalis, Loneliness and social dissatisfaction among behaviourally at-risk children, School Psychology International 29 (2008) 214-229.

[9] I. Buric, I. Soric, The role of test hope and hopelessness in self-regulated learning: Relations between volitional strategies, cognitive appraisals and academic achievement, Learning and Individual Differences 22 (2012) 523-529.

[10] H. Li, Y. Zhang, Factors predicting rural Chinese adolescents' anxieties, fears and depression, School Psychology International 29 (2008) 376-384.

[11] J. Dour, C.B. Cha, M.K. Nock, Evidence for an emotion cognition interaction in the statistical prediction of suicide attempts, Behaviour Research and Therapy 49 (2011) 294-298.

[12] I. Ajzen, The theory of planned behavior, Organizational Behaviour and Human Decision Processes 50(2) (1991) 179-211.

[13] C. Bonifield, C. Cole, Affective responses to service failure: Tức giận, hối tiếc, and retaliatory versus conciliatory responses. Marketing Letters 18(1-2) (2007) 85-99.

[14] S. Folkman, R.S. Lazarus, C. Dunkel-Schetter, A. DeLongis, R. J. Gruen. Dynamics of a stressful encounter: Cognitive appraisal, coping, and encounter outcomes. Journal of Personality and Social Psychology 50(5) (1986) 992-1003.

[15] S.Donoghue, H.M. de Klerk. Consumers' tức giận and coping strategies following appraisals of appliance failure. International Journal of Consumer Studies 37(2) (2013) 214-221.

[16] A.S. Mattila, H. Ro. Discrete negative emotions and customer dissatisfaction responses in a casual restaurant setting. Journal of Hospitality và Tourism Research 32(1) (2008) 89-107.

[17] R. Pekrun, The impact of emotions on learning and achievement: Towards a theory of cognitive/motivational mediators, Applied Psychology: An International Review 41 (1992) 359-376.

[18] F.T. Villavicencio, A.B.I.Bernardo. Negative Emotions Moderate the Relationship Between SelfEfficacy and Achievement of Filipino Students, Psychology Studies 58(3) (2013) 225-232.

[19] N. Stephens, K.P. Gwinner, Why don't some people complain? A cognitive-emotive process model of consumer than phiền behavior, Journal of the Academy of Marketing Science 26(3) (1998) 172-189.

[20] C.C.S. Brandenberger, G. Hagenauer, T. Hascher, Students' Self-Determined Motivation and Negative Emotions in Mathematics in Lower Secondary Education-Investigating Reciprocal 
Relations, Contemporary Educational Psychology, 2018, pp. 3-34.

[21] A.D. Rowe, J. Fitness, Understanding the Role of Negative Emotions in Adult Learning and Achievement: A Social Functional Perspective, Behavioral sciences 8(27) (2018) 1-20.

[22] J.A.D. Datu, Everyday discrimination, Negative emotions and academic achievement in Filipino secondary school students: Cross-sectional and cross-lagged panel investigations, Journal of School Psychology 68 (2018) 195-205.

[23] A. Mathur, G.P. Moschis, E. Lee, Stress and consumer behavior, Journal of Marketing Practice: Applied Marketing Science 5 (1999) 233-247.

[24] R. Bagozzi, Z. Gurhan-Canli, J. Priester, The social psychology of consumer behavior, New York: McGraw-Hill Education, 2002.
[25] I.J. Roseman, Appraisal determinants of discrete emotions, Cognition và Emotion 5(3) (1991) 161-200.

[26] D. Rai, I. Arroyo, L. Stephens, J.E. Beck, Repairing Deactivating Negative Emotions with Student Progress Pages, Springer-Verlag Berlin Heidelberg, 2013, pp. 795-786.

[27] J. Hair., M. Sarstedt., L. Hopkins., V. Kuppelwieser, Partial least squares structural equation modeling (PLS-SEM): An emerging tool in business research. European Business Review, 26(2) (2014) 106-121.

[28] C. Fornell, F.D. Larcker, Evaluating Structural Equation Models with Unobservable Variables and Measurement Error, Journal of Marketing Research 18 (1981) 39-50.

[29] J.C. Nunnally, I. Bernstein, The assessment of reliability, Psychometric Theory 3(1) (1994) 248-292. 